\title{
Generation of multipart images in the disconnected cerebral hemispheres
}

\author{
JUSTINE SERGENT \\ Montreal Neurological Institute, Montreal, Quebec, Canada \\ and \\ MICHAEL C. CORBALLIS \\ University of Auckland, Auckland, New Zealand
}

\begin{abstract}
We examined the respective capacities of the disconnected cerebral hemispheres of a commissurotomized subject to generate images of multipart objects. Two experiments were conducted, each comprising a perceptual and an imagery condition, in which the subject had to make a decision in response to segments of directly presented letters or to segments of imaged letters generated after presentation of their corresponding cases. In both experiments, the subject's two hemispheres performed with high accuracy on perceived and generated letters, and with equal efficiency on the generated letters. This indicated that there was no hemispheric asymmetry in the subject's creating and processing of images of multipart letters-a finding that is not consistent with results obtained with another "split-brain" patient. We therefore conclude that one should be cautious about basing theories of cerebral lateralization on data from this unusual neurological population.
\end{abstract}

Visual image generation is the process by which information stored in long-term memory is reactivated to give rise to a visual representation of an object's physical attributes, so that the object can be revisualized and inspected in the absence of direct sensory stimulation. Recent contributions to the study of mental imagery have been made by Kosslyn $(1987,1988)$ and Farah (1986), whose computational theory of visual cognition has led to the decomposition of the ability to revisualize images into a series of constituent operations. According to this theory, the generation component can be isolated as a single process, which the two cerebral hemispheres are not equipotent at implementing; only the left hemisphere (LH) is able to generate images of multipart objects (Farah, Gazzaniga, Holtzman, \& Kosslyn, 1985; Kosslyn, 1987). This suggestion is based on evidence from behavioral studies of normal subjects (Farah, 1986) and of commissurotomized patients (Farah et al., 1985; Kosslyn, Holtzman, Farah, \& Gazzaniga, 1985), but there are other reports that the right hemisphere (RH) does play a role in the generation of multipart images (Grossman, 1988; Morrow, Ratcliff, \& Johnston, 1985; Sergent, 1989).

Some of the best documented evidence in support of an exclusive role of the $\mathrm{LH}$ in the generation of multipart images comes from studies of callosotomized subjects, in experiments designed to isolate the generation component through a series of complementary tasks aimed at controlling for the lateralization of the other operations

Preparation of this paper was supported by the Medical Research Council of Canada. Correspondence should be addressed to Justine Sergent, Montreal Neurological Institute, 3801 University Street, Montreal, Quebec H3A 2B4, Canada. that constitute the generation task (Farah et al., 1985; Kosslyn et al., 1985). For instance, the callosotomized patient J.W. had to decide whether or not lowercase letters had segments extending above or below their main bodies in conditions in which the letters had to be generated from the presentation of their uppercase counterparts. J.W.'s LH performed this task perfectly, whereas his RH did no better than chance; control tasks showed that this could not be attributed to a failure to establish the correspondence between the lowercase and uppercase versions of a letter, or to an inability to make a decision about the lowercase letter. This suggested that the generation component per se was defective in J.W.'s RH (Farah et al., 1985). Additional evidence of an inability to generate letters was found in patient V.P. during the initial stages of the task (Kosslyn et al., 1985).

In the present study, we examined this issue further by testing another commissurotomized subject, L.B., who had already been tested in a series of visual imagery tasks such as mental rotation and image generation (Corballis \& Sergent, 1988; Sergent \& Corballis, 1989). Although L.B. had displayed strong evidence of RH superiority in tasks that required transformation of visual images, his performance on image generation tasks had thus far yielded ambiguous results from which no unequivocal conclusions could be drawn. In one task, L.B. was requested to imagine the position of the hands on a clock according to digitally presented times (e.g., 3:20; 9:05) and to decide whether the hands formed an angle greater or lesser than $90^{\circ}$. His LH performed significantly above chance $(67 \%)$, but his RH did not. This could point to an image generation deficit in the RH, but no control tasks were carried out to determine whether his $R H$ could read 
the digitally presented times and establish a correspondence between digital and analog times. In the other task, which was similar to that described earlier, opposite patterns of visual field asymmetry were obtained for the two dependent variables. Accuracy was significantly higher in LH (93.8\%) stimulation than in RH (82.3\%) stimulation, whereas latencies were significantly shorter in $\mathrm{RH}$ than in LH stimulations; there was thus a speed-accuracy tradeoff between the hemispheres, but none was found within each hemisphere. In addition, no control task was carried out, so it could not be determined whether the asymmetries reflected hemispheric difference at the generation level or in other operations involved in the task.

In this study, we sought new evidence of hemispheric competence at generating visual images, by including the control tasks required to isolate the generation component. In one experiment, L.B. decided whether or not lowercase letters, either directly presented or generated, had segments extending beyond their main bodies; in a second, he decided whether or not uppercase letters, either directly presented or generated, contained at least one curved segment.

\section{METHOD}

\section{Subject}

The subject was a 36-year-old commissurotomized patient, L.B., with radiologically verified section of the corpus callosum and anterior commissure. Details of his neurological and surgical history have been described elsewhere (e.g., Sergent, 1987).

\section{Equipment and Stimuli}

Two experiments were conducted 1 week apart, and different equipment was used in each one.

In the first experiment ("'extending segment"), the stimuli were displayed through a Gerbrands projection tachistoscope, with the same slides as those used in a similar study with normal subjects (Sergent, 1989); the letters subtended $3^{\circ}$ of visual angle and appeared $2.5^{\circ}$ to the right or left of a central fixation point. The stimuli were 16 letters, the lowercase version of which either had a part extending above or below the main body of the letter (b, d, f, g, h, p, q, t) or had no such part (a, $c, e, m, o, r, u, v)$. The lowercase versions were presented in the perceptual task and the uppercase version in the imagery task. Exposure duration was set at $150 \mathrm{msec}$, and the letters appeared as black on a white background. In the first part of this experiment, L.B. responded by moving one hand to the appropriate key, which was located $17 \mathrm{~cm}$ to the right or left of a central resting position. Because much time was spent making the response, the keys were later placed centrally, one ahead of the other on the midline axis, and L.B. responded with the index and middle fingers of one hand. The responding hand was counterbalanced within each part. Reaction times were measured from stimulus onset. The subject participated in 16 sessions of 32 trials each in the perceptual and the imagery conditions.

In the second experiment ("curved-straight segment"), all the letters of the alphabet except $I$ and $Z$ were used as stimuli. They were laterally $\left(2.3^{\circ}\right.$ eccentricity) presented on a fast-decay green phosphor screen (Model A2M2010) of an Apple Ile computer. Stimulus presentation was synchronized with the video frame rate $(60 \mathrm{~Hz})$ of the screen, and the stimuli were presented for 6 frames, corresponding to $100 \mathrm{msec}$. Prior to each stimulus presentation, a fixation dot appeared at the center of the screen. The response buttons were the " $N$ " and " $B$ " keys, which were located side by side at the middle of the bottom row of the computer keyboard. The subject responded with the index and middle fingers of the same hand, and the responding hand was counterbalanced across sessions. The task of the subject was to decide whether uppercase letters had a curved segment (e.g., B, P, S) or not (e.g., A, N, T). In the perceptual condition, uppercase letters were presented as stimuli, and, in the imagery condition, lowercase letters were presented and the subject had to image the corresponding uppercase letters and make his decision on the basis of this image. Eight sessions of 48 trials were carried out, equally distributed across conditions and responding hands.

\section{Procedure}

The procedure was the same in the two experiments. The subject was first requested to fixate the central point. After about $1 \mathrm{sec}$, a letter appeared in one of the visual fields. In the perceptual condition, the subject had to respond directly to each presented letter, and in the imagery condition, he had to image the corresponding case of each letter presented and make his decision on the basis of this image. Both speed and accuracy were stressed in the instructions, as well as the need to focus on the fixation point from the warning signal to response execution.

\section{RESULTS}

In both experiments, L.B. performed with high accuracy, and errors were evenly distributed across visual fields and conditions. No analysis of errors was therefore conducted. Percent correct responses and mean correct reaction times are presented in Table 1, for each task and condition as a function of visual field and responding hand.

\section{Extending Segment Task}

Correct reaction times were subjected to a 5-way analysis of variance, with the factors being mode of response (moving arm or finger), responding hand (left or right), visual field (left or right), condition (perceptual or imagery), and decision (extending segment or not). The effect of mode of response, which was highly significant $[F(1,96)=1,407]$, indicated faster finger- than armmovement responses. The main effect of visual field, which was significant $[F(1,96)=8.85, p<.005]$, resulted from faster responses to left than to right visual field presentations (964 and $997 \mathrm{msec}$, respectively). Also significant was the main effect of task; L.B.'s reaction times were faster in the perceptual $(922 \mathrm{msec})$ than in the imagery $(1,038 \mathrm{msec})$ condition $[F(1,96)=106.33]$.

Table 1

Mean Correct Reaction Times and Percent Correct Responses for Each Experiment

\begin{tabular}{lllll}
\hline \multirow{2}{*}{ Condition } & \multicolumn{2}{c}{ Left Hand } & & \multicolumn{2}{c}{ Right Hand } \\
\cline { 2 - 3 } & RT & \% Correct & RT & \% Correct \\
\hline
\end{tabular}

\begin{tabular}{ccccc}
$\begin{array}{c}\text { Perceptual } \\
\text { LVF }\end{array}$ & 856 & 95 & 943 & 95 \\
RVF & 951 & 97 & 942 & 96 \\
$\begin{array}{l}\text { Imagery } \\
\text { LVF }\end{array}$ & 1011 & 94 & 1047 & 96 \\
RVF & 1077 & 95 & 1021 & 93 \\
& & & \\
Perceptual & \multicolumn{5}{c}{ Curved-Stright Task } \\
LVF & 746 & 100 & 846 & 100 \\
RVF & 890 & 100 & 892 & 92 \\
Imagery & & & 1149 & 92 \\
LVF & 1601 & 92 & 1366 & 75 \\
RVF & 1348 & 92 & \\
\hline
\end{tabular}

Note-LVF = left visual field. RVF = right visual field. 
The main effect of responding hand was not significant, but it significantly interacted with condition and visual field. The interaction of hand and condition showed faster responses with the left than with the right hand in the perceptual condition (902 and $942 \mathrm{msec}$, respectively) and the reverse in the imagery condition $[1,043$ and $1,034$ msec, respectively; $F(1,96)=4.66, p<.05]$. More important is the interaction of visual field and hand $[F(1,96)=17.57, p<.01]$, which showed faster response of the left hand to the left field and of the right hand to the right field (see Table 1). This interaction was even more pronounced when the mode of response was taken into consideration, as was suggested by the 3-way interaction of mode of response, responding hand, and visual field $[F(1,96)=6.56, p<.05]$.

The critical interaction of condition and visual field was not reliable $[F(1,96)=1.53, p>.20]$, suggesting that the introduction of the image generation component did not differently influence the processing efficiency of the two hemispheres. However, the interaction of decision and visual field was significant $[F(1,96)=5.26$, $p<.05]$, and it showed that, whereas the two hemispheres were equally fast at deciding that a letter had an extending segment, the $\mathrm{RH}$ was faster than the $\mathrm{LH}$ at deciding that a letter had no extending segment. This occurred only in the perceptual condition; hence there was a significant 3-way interaction of condition, decision, and visual field $[F(1,96)=5.88, p<.05]$.

\section{Curved-Straight Task}

Correct reaction times were subjected to a 4-way analysis of variance, the factors being responding hand, visual field, condition, and decision. The main effect of condition was significant $[F(1,61)=50.37, p<.001]$, L.B. performing faster in the perceptual $(832 \mathrm{msec})$ than in the imagery $(1,366 \mathrm{msec})$ condition. The only other result to reach a reliable level of significance was the main effect of decision $[F(1,61)=6.99, p<.05]$, with curved uppercase letters being responded to faster than straight capital letters, whether the decision was made with respect to perceived letters or to their generated images. The interaction of visual field and condition was not significant $[F(1,61)=.36]$, indicating no different influence of the conditions on hemisphere processing.

\section{DISCUSSION}

In this study, we examined the respective capacity of the disconnected hemispheres of a commissurotomized subject to generate multipart images and to make decisions regarding parts of the images. The results showed that both hemispheres were capable of performing such a task, and the absence of communication between the two hemispheres of this patient suggests that this was achieved through each hemisphere's operating on its own. This finding confirms earlier evidence from normal subjects (Sergent, 1989) and from this patient (Corballis \& Sergent, 1988) that both cerebral hemispheres have the capacity to generate multipart images, and it departs from evidence obtained from another patient (J.W., Farah et al., 1985; Kosslyn et al., 1985) that the right hemisphere cannot create images of multipart objects.
The higher accuracy of the LH than of the RH that was previously observed by Corballis and Sergent (1988) did not obtain in the present experiment, perhaps because the larger stimuli may have facilitated the early encoding stages and thus given some processing advantage to the RH (e.g., see Sergent, 1983). Nonetheless, the absence of a significant interaction between visual field and condition in the two experiments suggests that this factor influenced the perceptual stages and did not interfere with the imagery component of the imagery condition. Even though the design of the two experiments does not permit the isolation of the generation component as such from the operations subsequently carried out on the images of the generated letters, the particular neurological condition of the patient ensured that the generation process was carried out within each hemisphere. It thus seems reasonable to infer that both hemispheres of this patient have the capacity to generate images of multipart letters.

The significance of the present finding for the understanding of the contribution of the normal cerebral hemispheres to image generation is obviously limited, since much caution is required in generalizing from results of commissurotomized patients to the general population. It is, however, noteworthy that L.B.'s performance differed from that of J.W. (Kosslyn et al., 1985), who was unable to generate images of multipart objects. This divergence illustrates the idiosyncrasies of "split-brain" subjects, as well as the risk one runs in basing theories on their performance. Yet the view that the RH is unable to generate multipart images, as part of Kosslyn's $(1987,1988)$ theory, is based solely on findings from J.W., for neither normal nor brain-damaged subjects have so far been tested in experiments that address this issue (see Sergent, in press, for a review). Had Kosslyn tested L.B. rather than J.W., a different theory might have emerged, though not necessarily a more justified one.

\section{REFERENCES}

Corballis, M. C., Sergent, J. (1988). Imagery in a commissurotomized patient. Neuropsychologia, 26, 13-26.

FARAH, M. J. (1986). The laterality of mental image generation: A test with normal subjects. Neuropsychologia, 24, 541-544.

Farah, M. J., Gazzaniga, M. S., Holtzman, J. D., Kosslyn, S. M. (1985). A left hemisphere basis for mental imagery? Neuropsychologia, 23, 115-118.

Grossman, M. (1988). Drawing deficits in brain-damaged patients' freehand pictures. Brain \& Cognition, 8, 189-205.

KossLyN, S. M. (1987). Seeing and imaging in the two cerebral hemispheres: A computational approach. Psychological Review, 94, 148-175.

Kosslyn, S. M. (1988). Aspects of a cognitive neuroscience of mental imagery. Science, 240, 1621-1626.

Kosslyn, S. M., Holtzman, J. D., Farah, M. J., Gazzaniga, M. S. (1985). A computational analysis of mental image generation: Evidence from functional dissociations in split-brain patients. Journal of Experimental Psychology: General, 114, 311-341.

MorRow, L., RATCLIFF, G., \& Johnston, C. S. (1985). Externalising spatial knowledge in patients with right hemisphere lesions. Cognitive Neuropsychology, 2, 265-273.

Sergent, J. (1983). The role of the input in visual field asymmetries. Psychological Bulletin, 93, 481-512.

SERGENT, J. (1987). A new look at the human split brain. Brain, 110, 1375-1392.

SERGENT, J. (1989). Image generation and processing of generated images in the cerebral hemispheres. Journal of Experimental Psychology: Human Perception \& Performance, 15, 170-178.

SERGENT, J. (in press). The neuropsychology of image generation: Data, method, and theory. Brain \& Cognition.

Sergent, J., \& Corballus, M. C. (1989). Categorization of disoriented faces in the cerebral hemispheres of normal and commissurotomized subjects. Journal of Experimental Psychology: Human Perception \& Performance, 15, 701-710.

(Manuscript received December 14, 1989.) 